\title{
Performance Analysis and Tube Inlet Orifice Length Evaluation of a Once-Through Steam Generator
}

\author{
Hun Sik Han ${ }^{1, *}$, Han-Ok Kang ${ }^{1}$, Juhyeon Yoon ${ }^{1}$, Young In $\mathrm{Kim}^{1}$, Youngmin Bae ${ }^{1}$, and Sang Ji Kim ${ }^{1}$ \\ ${ }^{1}$ Korea Atomic Energy Research Institute, 34057 Daejeon, South Korea
}

\begin{abstract}
A numerical study is conducted for performance analysis and secondary side screw-type tube inlet orifice design of a once-through steam generator (OTSG). Various tube plugging conditions and power levels are considered, and the secondary coolant flow rate is adjusted to maintain a constant thermal power. Comprehensive numerical solutions are acquired to evaluate the OTSG thermal-hydraulic performance and minimum orifice length under various operating conditions. The OTSG performance is analyzed according to the tube plugging condition in terms of the OTSG thermal power, steam outlet superheat degree, and secondary coolant pressure drop. The results obtained show that a constant thermal power can be maintained by properly adjusting the secondary coolant flow rate with a variation of the steam outlet superheat degree and secondary coolant pressure drop when the OTSG operates at high power level. The required minimum orifice length to suppress the flow oscillation below the allowable level is evaluated. The lowest power level results in the highest minimum orifice length, and non-plugging condition provides a limiting case for the orifice length criterion.
\end{abstract}

\section{Introduction}

An integral-type pressurized water reactor employs once-through steam generators (OTSGs) owing to its advantages in compactness and simplicity of flow path arrangement [1]. Figure 1 shows the exterior view of an OTSG using helically coiled tubes, as considered in this study. In general, the OTSG operates under counter flow conditions. The primary coolant flows down across the helically coiled tube bundle, and the secondary feedwater flows up through the helically coiled tubes. The primary coolant flows outside the tubes and releases thermal energy into the secondary side to produce steam. The secondary feedwater inside the tube absorbs heat

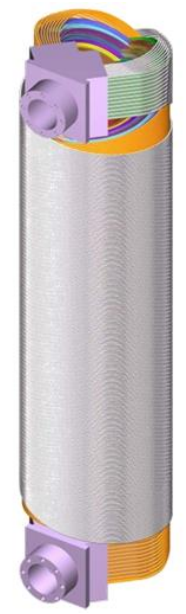

Fig. 1. Exterior view of the helically coiled tube. from the primary coolant and changes to superheated steam.

Such a phase change of the secondary feedwater brings forth density-wave oscillations, which lead to flow oscillations. These take place as a result of phase lag and feedback among flow rate, pressure drop, and phase-change processes. Increasing the system pressure, and increasing the inlet hydraulic resistance in particular, are stabilizing, whereas increasing the outlet hydraulic resistance and increasing the pressure loss in the twophase region are destabilizing [2]. Therefore, because such density-wave oscillations are suppressed by the strong stabilizing effect of the added single-phase resistance, it is necessary to install an orifice inside the tube at the entrance region for flow stabilization [3].

For this reason, a screw-type tube inlet orifice was introduced and detailed orifice design was implemented for the MRX OTSG (JAERI-developed integral reactor [4]. In Reference 4, the secondary coolant outlet pressure was controlled to maintain constant thermal power of the OTSG and the corresponding orifice length was evaluated. Instead of the secondary coolant outlet pressure control, however, another operation strategy can be applied to maintain constant thermal power. In this paper, the secondary coolant flow rate is adjusted for constant thermal power operation and the corresponding orifice length is evaluated.

\section{Analysis method}

\subsection{OTSG performance}

\footnotetext{
* Corresponding author: hunsikhan@gmail.com
} 
To evaluate the thermal-hydraulic performance of such a helically coiled tube OTSG, a well-established numerical code, ONCESG, is used. The ONCESG code was developed at the Korea Atomic Energy Research Institute (KAERI) for thermal-hydraulic design and performance analysis of OTSGs using helically coiled tubes [1]. In the present simulation, the friction factors and heat transfer coefficients are calculated using SKBK correlations for both the tube side and shell side of the helical tubes.

In the thermal-hydraulic design, a design margin is added to the initial clean design to compensate for design uncertainties and performance deterioration by tube plugging [5]. Here, the design margins for uncertainties and tube plugging are taken into account by applying an area utilization factor in each control volume and by changing the number of tubes. In the present study, the design data of MRX OTSG [4] is used, and the thermal-hydraulic performance of the OTSG is investigated in terms of the thermal power, outlet degree of superheat, and secondary coolant pressure drop. The steam generator condition is divided into five steps according to the plugging ratio, as defined in Table 1 . Here, SG condition-1 represents the initial stage of the OTSG operation without tube plugging, whereas SG condition-5 denotes the final stage when the OTSG experiences tube plugging of $\alpha=10.3 \%$. The average tube length of the OTSG is $47.0 \mathrm{~m}$ with a tube plugging ratio of $\alpha=10.3 \%$ and $5 \%$ design uncertainty $(a=0.95)$ [4].

\subsection{Tube inlet orifice}

Consider a screw-type tube inlet orifice, as sketched in Figure 2. The orifice has a narrow spiral flow channel that provides high flow resistance. Introducing the hydraulic resistance ratio, orifice pressure drop, and geometry formulas gives an orifice length criterion for flow stabilization as follows [4]:

$$
L_{\text {ori }} \geq L_{\text {ori_min }}=\frac{K_{\text {ori_min }} \rho_{\mathrm{e}} v_{\mathrm{e}}^{2}-K_{\mathrm{i}} \rho_{\mathrm{i}} v_{\mathrm{c} \_1}{ }^{2}-K_{\mathrm{e}} \rho_{\mathrm{c} \_2} v_{\mathrm{c} \_2}{ }^{2}}{\left[f / w+0.1 /\left(\pi D_{\text {avg }}\right)\right] \rho_{\mathrm{c}_{-} 1} v_{\mathrm{c}_{\_} 1}{ }^{2}} \sin \theta .
$$

Here, $L_{\text {ori_min }}$ is the minimum orifice length for suppression of the flow oscillation below the allowable level, $K_{\text {ori min }}$ is the minimum orifice loss coefficient, $K_{\mathrm{i}}$ is the inlet loss coefficient, $K_{\mathrm{e}}$ is the exit loss coefficient, $w$ is the orifice channel width, $\theta$ is the incline angle of the orifice channel, $D_{\text {avg }}$ is the average coiling diameter of the orifice channel, $v_{\mathrm{c}}$ is the cross-sectional average velocity in the orifice channel, and $f$ is the friction factor. The subscripts 1 and 2 denote the beginning and end of the orifice channel, respectively.

Table 1. Steam generator conditions

\begin{tabular}{|c|c|c|c|c|c|}
\hline SG condition & 1 & 2 & 3 & 4 & 5 \\
\hline Plugging ratio, $\alpha[\%]$ & 0.0 & 2.6 & 5.2 & 7.7 & 10.3 \\
\hline Tube no., $N[\mathrm{e}]$ & 388 & 378 & 368 & 358 & 348 \\
\hline
\end{tabular}

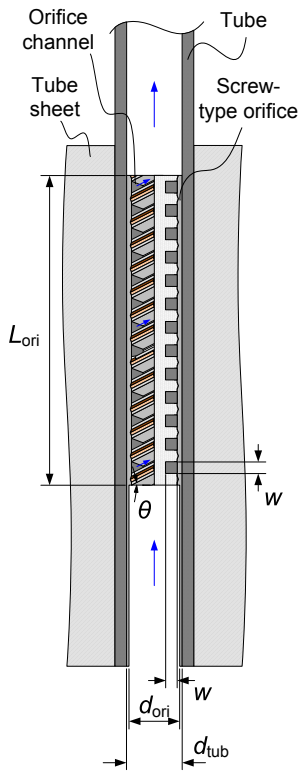

Fig. 2. Configuration of screw-type tube inlet orifice

In the present study, the minimum hydraulic resistance of $\kappa_{\min }=1.92$, orifice diameter of $d_{\text {ori }}=14.8$ $\mathrm{mm}$, and surface roughness of $\delta=0.01 \mathrm{~mm}$ are applied to the proposed design of the OTSG secondary side tube inlet orifice, and the orifice length is calculated at various power levels and tube plugging conditions.

\section{Results and discussion}

\subsection{OTSG performance}

The performance variation according to the secondary coolant flow rate $m$ at high power level is plotted in Figure 3a. The thermal power almost linearly increases with the secondary coolant flow rate. It is evident that SG condition-1 gives a higher thermal power compared to SG condition-5. This means that the OTSG performance deteriorates as it experiences a heat transfer area reduction by flow path blockage due to tube plugging. It is also noticeable that the green square symbol in Figure 3a represents the design point because the OTSG was originally designed with a design margin that considered a tube plugging of $\alpha=10.3 \%$. When the OTSG begins its first operation without tube plugging, however, the operating point is located on the red line of SG condition-1. Thus, the OTSG will show a higher performance compared to the original design at the given secondary coolant flow rate (see the red triangular symbol in Figure 3a). Such unintended OTSG operation is unsuitable for stable operation of the primary system. For this reason, it is necessary to operate the OTSG with properly reduced secondary coolant flow rate at the early stage of plant operation. This initial operating point is presented in Figure $3 \mathrm{a}$ with a red circular symbol. Consequently, the OTSG can maintain thermal power during plant lifetime by gradually increasing the secondary coolant flow rate.

The degree of superheat at the tube outlet decreases with increasing of the secondary coolant flow rate, as 


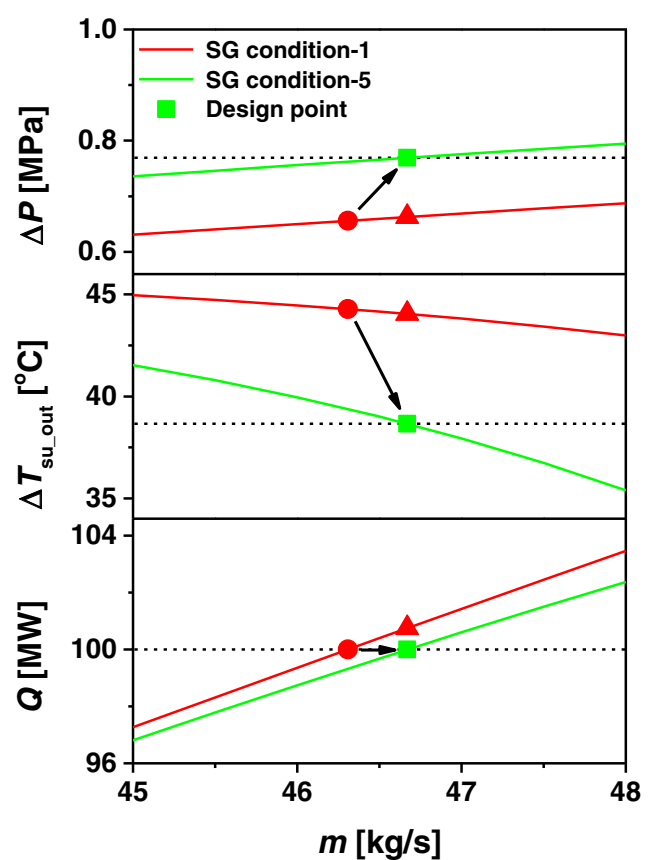

(a)

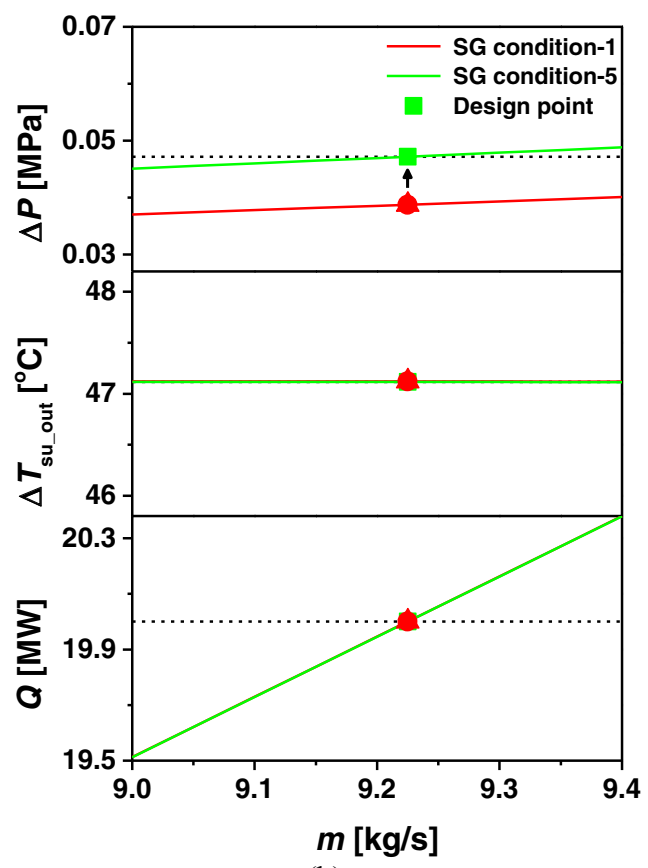

(b)

Fig. 3. Performance variation according to secondary coolant flow rate: (a) high power and (b) low power

shown in Figure 3a. SG condition-1 results in a relatively gentle decline of the outlet degree of superheat compared to SG condition-5 because SG condition-1 provides significant potential to transfer heat energy. Thus, it is possible to maintain the secondary coolant outlet temperature relatively close to the primary coolant inlet temperature when the secondary coolant flow rate is elevated. From a long-term operation point of view, the steam outlet superheat degree decreases during plant lifetime if the secondary coolant flow rate is controlled for a constant thermal power, as shown in Figure 3a. Figure $3 \mathrm{a}$ also shows a linear increment in the secondary coolant pressure drop with an increase in the secondary coolant flow rate. SG condition-5 results in a higher secondary coolant pressure drop compared to SG condition-1 owing to the higher flow resistance induced by tube plugging.

The low power operation shows the same trend as the high power level except for the outlet degree of superheat, as presented in Figure 3b. The outlet degree of superheat is almost unchanged with respect to the secondary coolant flow rate because the OTSG provides an exceedingly sufficient heat transfer area in transferring a small amount of heat energy and this causes the secondary coolant outlet temperature to reach the primary coolant inlet temperature. For the same reason, the thermal performance is almost identical regardless of the tube plugging condition. Thus, it is unnecessary to adjust the secondary coolant flow rate. The secondary coolant pressure drop increases as the steam generator condition changes from 1 to 5 due to the increased flow resistance by the tube plugging.

\subsection{Tube inlet orifice}

Figure 4 shows the variation of the minimum orifice length $L_{\text {ori min }}$ according to the tube plugging condition when the screw-type tube inlet orifice has a channel geometry of $w=3.0 \mathrm{~mm}$ and $\theta=12^{\circ}$. The minimum orifice length ranges from $183.8 \mathrm{~mm}$ to $198.9 \mathrm{~mm}$ for $100 \%$ power level, and from $285.1 \mathrm{~mm}$ to $288.0 \mathrm{~mm}$ for $20 \%$ power level. As expected, the low power level results in a higher minimum orifice length because flow instability tends to be more severe at a low feedwater flow rate [6] and this accordingly brings forth a higher orifice loss coefficient [4]. Thus, the secondary coolant flow rate for the minimum power level should be chosen as the orifice design condition; this implies that the orifice length is determined by the lowest power level considered in plant power operation.

Each power level has its own highest minimum orifice length at the non-plugging condition because this operating condition yields the lowest secondary coolant flow rate per tube. A minimum orifice length of $L_{\text {ori_min }}=$ $285.1 \mathrm{~mm}$ can be selected if the lowest power level is $20 \%$ and the steam generator condition is 5 , which means 40 tubes are plugged in. However, the OTSG can operate under $0 \%$ tube plugging condition, which requires a minimum orifice length of $L_{\text {ori_min }}=288.0 \mathrm{~mm}$.

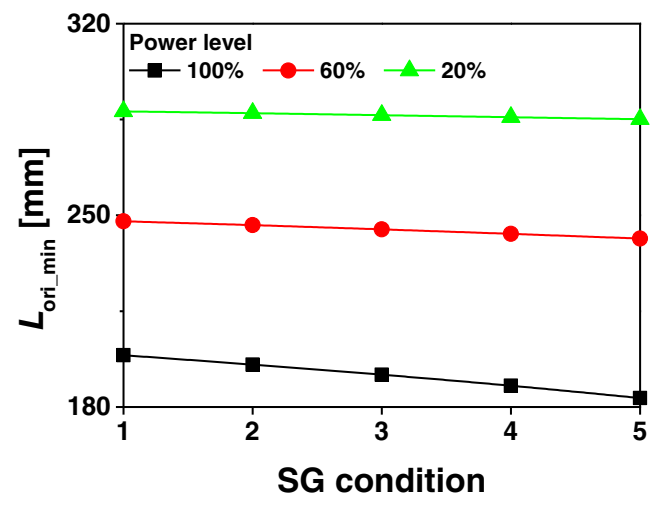

Fig. 4. Minimum orifice length according to tube plugging condition $\left(w=3.0 \mathrm{~mm}\right.$ and $\left.\theta=12^{\circ}\right)$ 
The orifice length should be higher than the minimum orifice length (see equation (1)), and thus the required minimum orifice length to limit the flow oscillations to below the allowable level regardless of the operating condition should be the highest one. This means the orifice length should be at least $288.0 \mathrm{~mm}$ (i.e., $L_{\text {ori }} \geq$ $288.0 \mathrm{~mm}$ ) to ensure stable power operation at power levels of $20 \%-100 \%$.

\section{Conclusions}

The effect of tube plugging on the thermal-hydraulic performance of an OTSG and on screw-type tube inlet orifice design has been investigated. The steam generator condition is divided into five steps according to the tube plugging ratio, and the OTSG performance is analyzed in terms of the OTSG thermal power, steam outlet superheat degree, and secondary coolant pressure drop. The results reveal that long-term constant full power operation can be achievable by properly elevating the secondary coolant flow rate, at the expense of decreased steam outlet superheat degree and increased secondary coolant pressure drop, as the OTSG suffers from a gradual deterioration in performance due to tube plugging. For low power operation, however, it is not necessary to adjust the secondary coolant flow rate because it is almost unchanged according to the tube plugging condition. The minimum orifice length is evaluated using the thermal-hydraulic analysis results of the OTSG and the orifice length criterion. The results obtained show that the lowest power level in plant power operation with non-plugging condition provides a limiting case for the orifice length determination. To ensure stable operation in a power range of $20 \%-100 \%$, the orifice length should be at least $288.0 \mathrm{~mm}$ (i.e., $L_{\text {ori }} \geq$ $288.0 \mathrm{~mm}$ ) when the orifice has a channel geometry of $w$ $=3.0 \mathrm{~mm}$ and $\theta=12^{\circ}$.

This work was supported by the National Research Foundation of KOREA (NRF) funded by the Ministry of Science and ICT (No. NRF-2018M2A8A4081307), South Korea.

\section{References}

1. J. Yoon, J.-P. Kim, H.-Y. Kim, D.J. Lee, M.H. Chang, J. Nucl. Sci. Technol., 37, 445-454 (2000)

2. S.M. Ghiaasiaan, Two-Phase, Boiling and Condensation in Conventional and Miniature Systems (Cambridge University Press, New York, 2008)

3. R.T. Lahey, F.J. Moody, The Thermal-Hydraulics of a Boiling Water Nuclear Reactor (Second Edition, American Nuclear Society, USA, 1996)

4. H.S. Han, R. Alshehri, H.-O. Kang, J. Yoon, Y.I. Kim, S.J. Kim, J. Mech. Sci. Tehonol., 33, 38413849 (2019)

5. R.L. Shilling, Heat Transfer Engineering, 33, 10941104 (2012)

6. H.-O. Kang, J.K. Seo, Y.W. Kim, J. Yoon, K.K. Kim, J. Nucl. Sci. Technol., 44, 64-72 (2007) 